\title{
Adult Glioblastoma and the Role of DAXX (Death Domain Associated Protein) Tumor Biomarker
}

\author{
Sean M Hacking ${ }^{1 *}$, Cao Jin ${ }^{1}$, Seungjun Ahn ${ }^{2}$, Deepika Savant ${ }^{1}$ and Mansoor Nasim ${ }^{1}$ \\ ${ }^{1}$ Department of Pathology and Laboratory Medicine, Zucker School of Medicine at Hofstra Northwell, USA \\ ${ }^{2}$ Department of Biostatistics, Zucker School of Medicine at Hofstra Northwell, USA
}

*Corresponding author: Sean M Hacking, MB, BCh, BAO, Department of Pathology and Laboratory Medicine, Zucker School of Medicine at Hofstra Northwell, USA

\section{Introduction}

Glioblastoma, also known as glioblastoma multiforme (GBM), is the most aggressive primary brain cancer [1]. GBM comprises $16 \%$ of all primary brain neoplasms with an incidence rate of 3.2 per 100,000 people [1]. Patients with GBM have a poor prognosis, less than $5 \%$ of them survive for more than 5 years [1]. In the treatment of GBM there is currently insufficient information to improve disease management because conventional treatments universally fail $[1,2]$. Currently there is a crucial need to identify relevant targets in GBM in order to design new therapeutic agents $[1,2]$.

In children brain tumors are currently the leading cause of cancer-related mortality and morbidity as they are the most common solid tumors [3]. Histologically GBMs presenting in children are the same as there adult counterparts (per world health organization grading system), however in pediatric populations loss of death domain-associated protein (DAXX) has been shown to suppress tumor growth and increases survival time $[4,5]$. DAXX was originally characterized as a Fas death receptor binding protein, however more recently DAXX has also been found to interact with ATP-dependent helicase ATRX (ATRX) involving the histone chaperone complex $[6,7]$. More specifically DAXX acts as a histone chaperone protein that directly interacts with H3.3 (a conserved histone variant that is structurally very close to the canonical histone, $\mathrm{H} 3$ ) and facilitates its deposition on chromatin [4]. Therefore, DAXX restores H3.3, impeding oncogene expression [4].
It has been definitively established in pediatric research studies that DAXX negatively regulates genes involved in oncogenesis [4]. With genome sequencing identifying mutations in the H3.3-ATRX-DAXX chromatin remodeling pathway in $44 \%$ of the pediatric GBM tumors [8]. However, there has been limited research examining the role of DAXX in adult GBM. No large studies have identified DAXX mutations or protein expression in an adult GBM cohort. This could be because genetic alterations of DAXX are seen to be uncommon in adult GBM. It is possible that DAXX may play a role in adult GBM tumors in a similar manner to that established in pediatric models.

\section{Materials and Methods}

\section{Design}

This study is a retrospective study of the histopathology and the biomarker expression of the patients who were diagnosed with Glioblastoma at the department of pathology and laboratory medicine, Zucker School of Medicine at Hofstra Northwell. 72 cases of GBM were included in our study. An immunohistochemical method for DAXX determination (Anti-DAXX) was performed to determine protein expression. Factors including MRI size and survival status were compared to different cohorts of Daxx-1 expression. These cohorts include limited expression $<25 \%$ and definite expression (> 25\%) which were quantified according to overall, cytoplasmic and nuclear staining percentages.

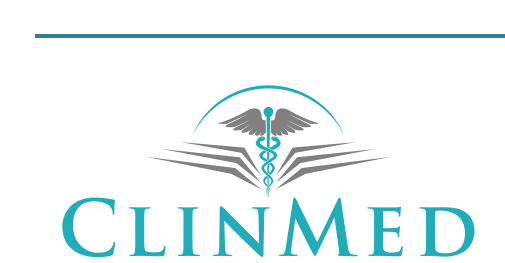

INTERNATIONAL LIBRARY

Citation: Hacking SM, Jin C, Ahn S, Savant D, Nasim M (2019) Adult Glioblastoma and the Role of DAXX (Death Domain Associated Protein) Tumor Biomarker. Int J Pathol Clin Res 5:089. doi. org/10.23937/2469-5807/1510089

Accepted: May 16, 2019: Published: May 18, 2019

Copyright: (C) 2019 Hacking SM, et al. This is an open-access article distributed under the terms of the Creative Commons Attribution License, which permits unrestricted use, distribution, and reproduction in any medium, provided the original author and source are credited. 


\section{Case selection}

The electronic database at the department of pathology and laboratory medicine, Zucker School of Medicine at Hofstra Northwell was searched for patients diagnosed with GBM between 2007 and 2017. Seventy two archived cases of GBM, all received between 2007 and 2017, were retrieved and reviewed. Data from the

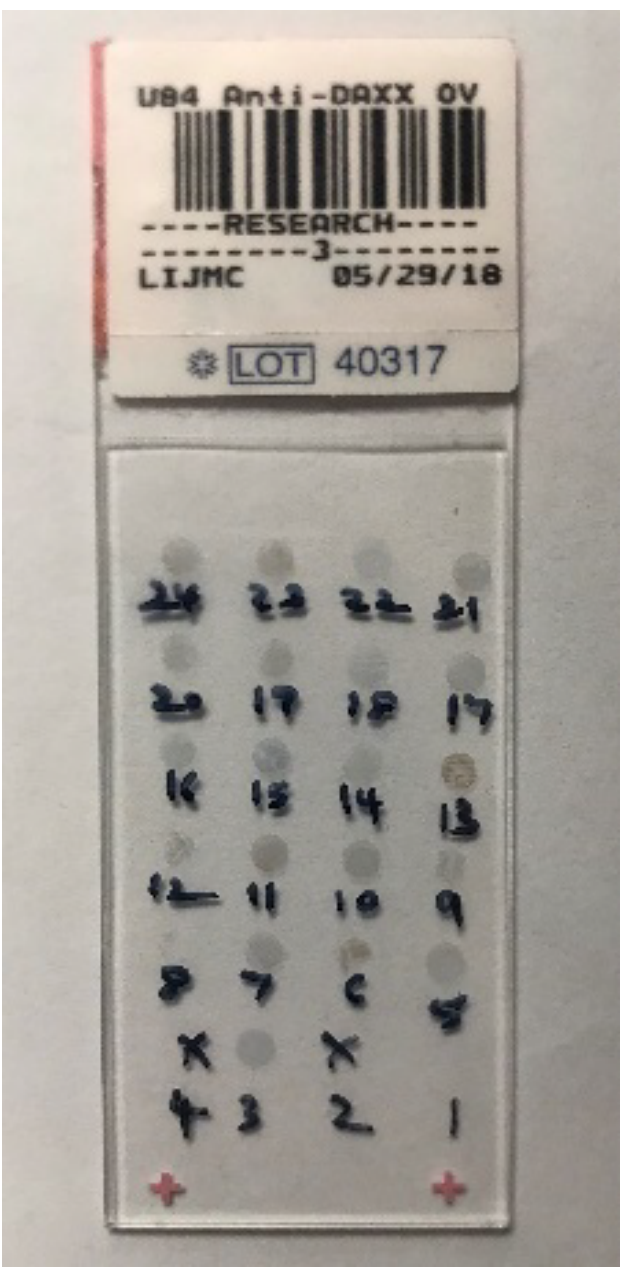

Figure 1: Tissue microarrays (TMA). cancer registry and electronic data base was used in order to assign tumor size and survival status to our cases. All samples were obtained with informed consent after approval of the Institutional Review Board of the respective hospitals they were treated in and were independently reviewed by a senior neuropathologist and a pathology resident according to the World Health Organization guidelines.

\section{Tissue microarray}

Tissue microarrays (TMA) are paraffin blocks produced by extracting small tissue cores from different paraffin donor blocks and re-embedding these into a single recipient (microarray) block at defined array coordinates [9]. Tissue microarrays were constructed from the 72 GBM cases. This was accomplished by retrieving identical tissue cores (1.5 mm diameter) sampled from the most representative areas of each patients donor blocks. In our study only viable tumor tissue was extracted for tissue cores and areas with signifiant hemmorhage and necrosis were not sampled. The paraffin blocks were then cut and placed onto slides (Figure 1). A map was created in order to identify each patient's tumor (site on the TMA), and patient tumors were labeled 1-24. Each TMA (block and slide) was individually labeled and two cores were left blank in every TMA in order to maintain site specific orientation. In our study and as experienced in other settings we found TMA to be an extremely practical and cost effective tool for immunohistochemical analysis of tissues [9].

\section{Immunohistochemical analysis}

Immunohistochemical stains for DAXX (Anti-DAXX (Sigma Life Science; HPA008736, 1:100)) was used on the tissue microarrays we created (Figure 2). DAXX expression was semi-quantified. Tumors with absent DAXX expression were required to have positive internal controls (normal endothelial, stromal or inflammatory cells)
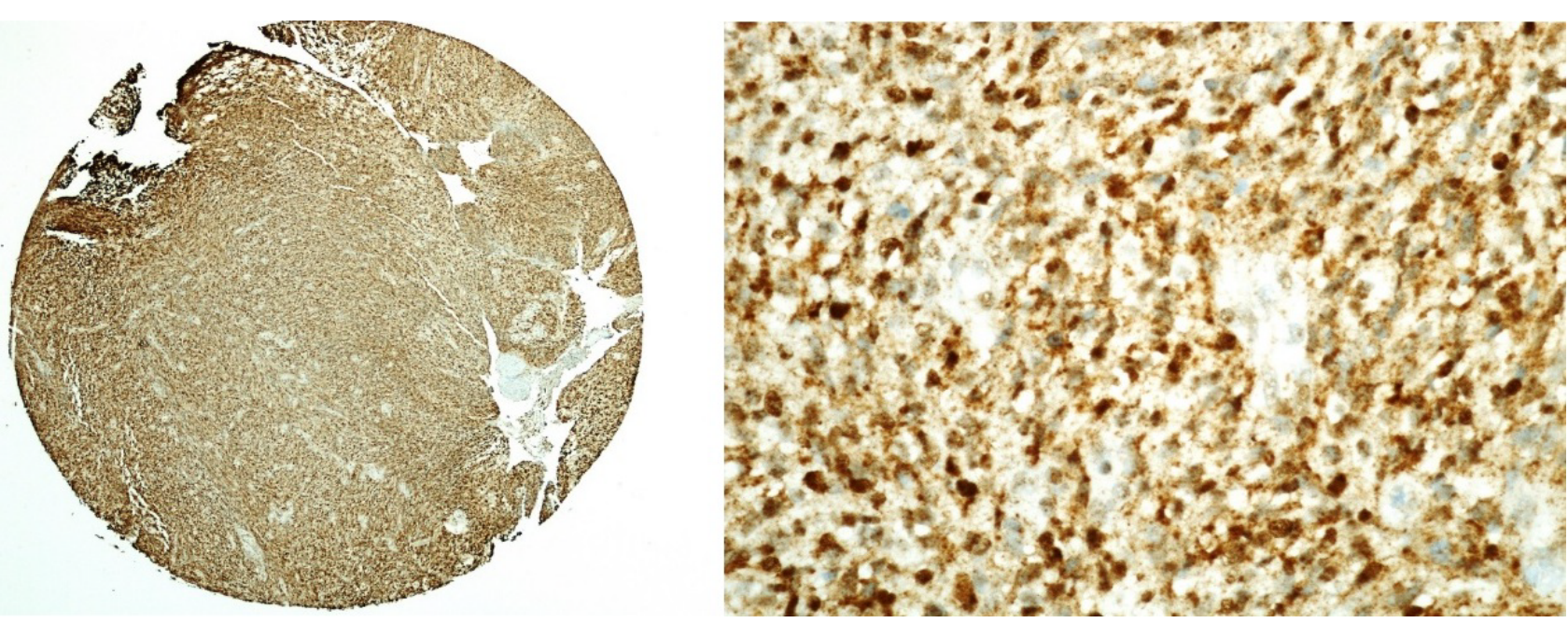

Figure 2: Anti-DAXX (Sigma Life Science; HPA008736, 1:100).

Left: Low power $(2 x)$ showing $1.5 \mathrm{~mm}$ diameter tissue core stained with DAXX; Right: High power (40x) showing DAXX staining pattern of GBM tumor cells. 
before being considered negative. All the staining was performed at the Immunopathology Laboratory of Long Island Jewish Medical Center (Northwell Health System, New Hyde Park, NY). With regards to DAXX expression tumors tended to have either limited expression (less that $15-20 \%$ staining) or definitive expression (greater that $30-35 \%$ staining). As a result of these findings we decided to use a cutoff value of $25 \%$ (moderate intensity staining). The staining was categorized as definitive expression if $25 \%$ or more of the tumor cells showed moderate intensity staining. Staining was categorized as limited expression if less than $25 \%$ of the tumor cells showed moderate intensity staining.

\section{Statistical analysis}

The Kaplan-Meier product limit method was used to estimate the survival time in days from date of diagnosis, and the groups ( $<25 \%$ vs. $\geq 25 \%$ ) were compared using the log-rank test for each DAXX cohort variables. The Wilcoxon signed-rank test was utilized for paired analysis of DAXX data. The differences were calculated as overall minus each of the two DAXX cohort variables (i.e. overall minus nuclear, and overall minus cytoplasm). The two-sample t-test was utilized for comparing tumor size at diagnosis between groups (< $25 \%$ vs. $\geq 25 \%$ ) for each DAXX cohort variables. Results were considered statistically significant if $p<0.05$. Analysis was conducted using SAS v. 9.4 (SAS Institute,
Inc., Cary, NC).

\section{Results}

\section{Spectrum of staining patterns in GBM}

Our study found a wide spectrum of DAXX staining in our 72 GBM cases. The mean for cytoplasmic staining percentage was $25 \%$ and the mean for nuclear staining percentage was $22 \%$. The overall staining percentage was highest with $30.26 \%$ of cells showing at least moderate intensity staining. For the cytoplasmic staining cohort 45 patients had under $25 \%$ staining and 27 patients had greater or equal to $25 \%$ staining. For the nuclear staining cohort 48 patients had under $25 \%$ staining and 24 patients had greater or equal to $25 \%$ staining. Finally for the overall staining cohort 39 patients had under $25 \%$ staining and 33 patients had greater or equal to $25 \%$ staining.

\section{Tumor size between groups ( $<25 \%$ vs. $\geq 25 \%$ ) for each DAXX cohort variables}

Statistical analysis for tumor size between definite and limited expression of Anti DAXX stain was statistically significant. Patients who had less than $25 \%$ cytoplasmic staining had significantly larger tumor size compared to patients who had greater than or equal to $25 \%$ cytoplasmic staining (Mean (SD) = 3.65 (1.75) vs. 2.77 (1.56), p < 0.036), (Figure 3). Patients who had less than



Figure 3: Boxplot of tumor size (Cytoplasmic DAXX staining cohort). 
$25 \%$ nuclear staining had significantly larger tumor size compared to patients who had greater than or equal to $25 \%$ staining (Mean (SD) = 3.64 (1.61) vs. 2.68 (1.81), p $<0.026)$, (Figure 4). Finally patients who had less than $25 \%$ overall staining had significantly larger tumor size compared to patients who had greater than or equal to $25 \%$ overall staining (Mean (SD) $=3.79$ (1.64) vs. 2.76 (1.67), $\mathrm{p}<0.010)$, (Figure 5).

\section{Kaplan-Meier product-limit analysis}

Kaplan-Meier product-limit analysis for prognosis between definite and limited expression of Anti DAXX stain was not statistically significant. There was no statistically significant difference in survival time for the cytoplasmic staining cohort $(p<0.915)$. The estimated median time to survival for patients who had less than

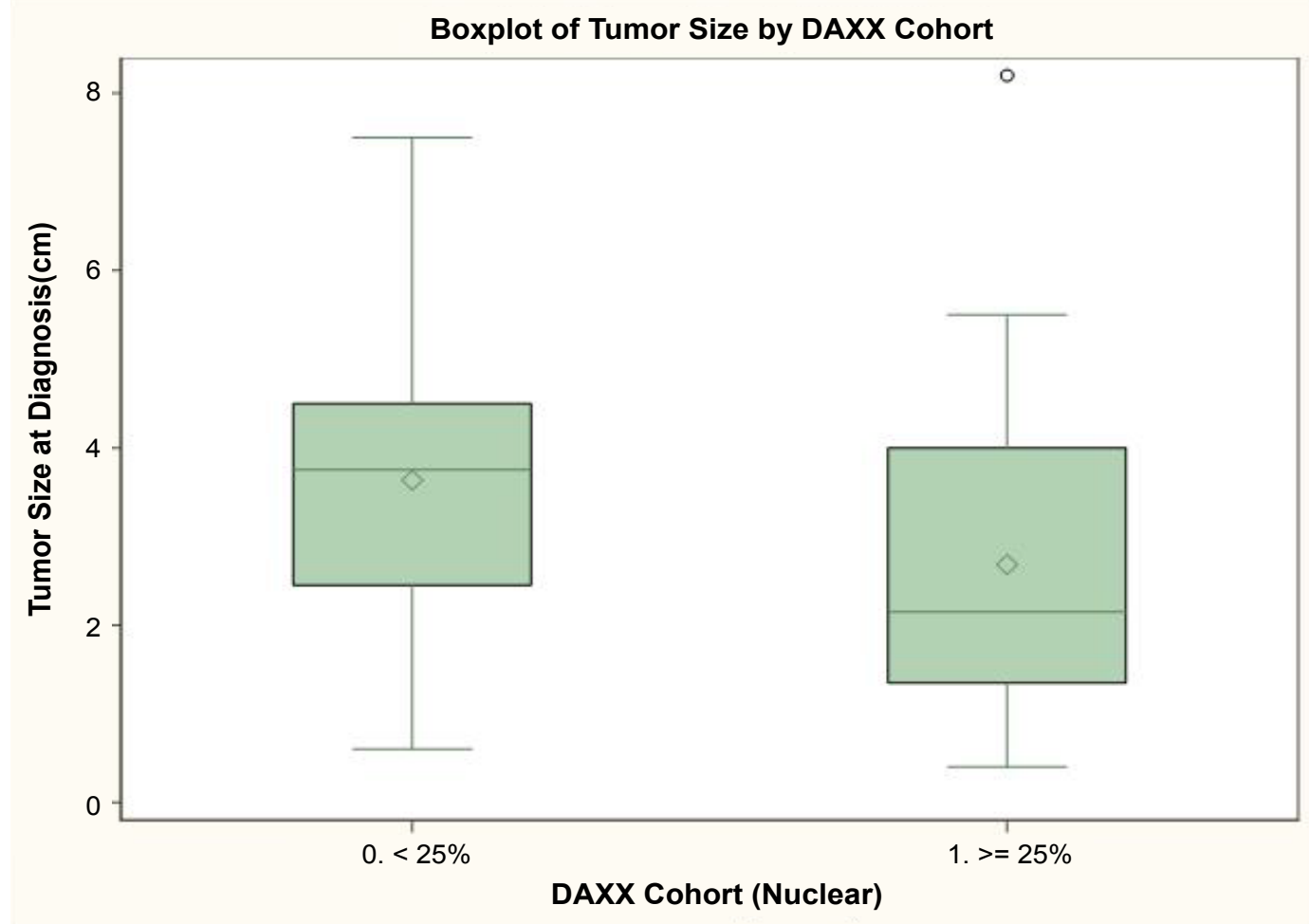

Figure 4: Boxplot of tumor size (Nuclear DAXX staining cohort).

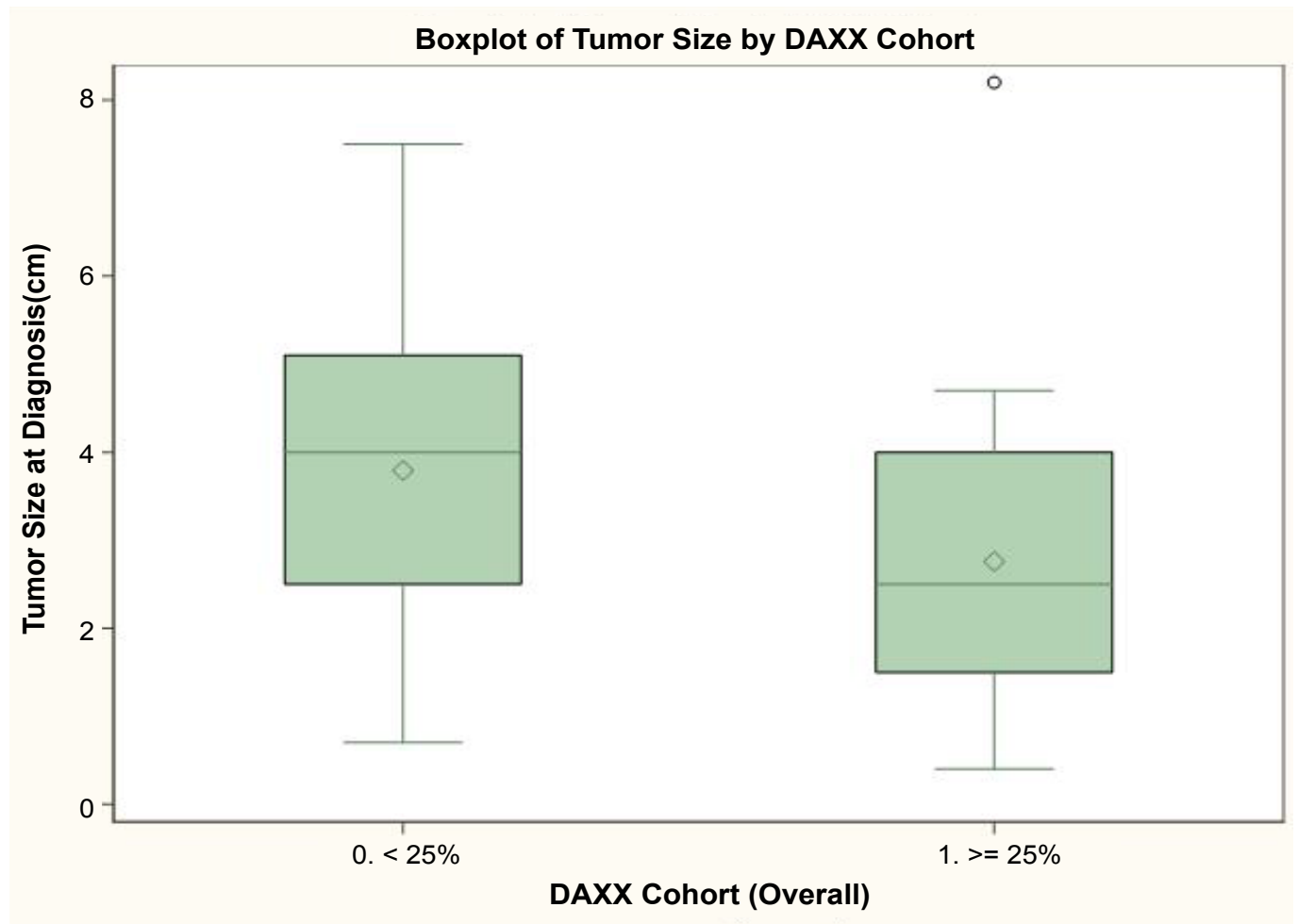

Figure 5: Boxplot of tumor size (Overall DAXX staining cohort). 
25\% cytoplasmic staining was 385 days $(95 \% \mathrm{Cl}: 206$ days, 511 days), and for patients who had greater than or equal to $25 \%$ cytoplasmic staining was 483 days (95\% Cl: 218 days, 635 days). There was no statistically significant difference in survival time for the nuclear staining cohort $(p<0.803)$. The estimated median time to survival for patients who had less than $25 \%$ nuclear staining was 460 days (95\% Cl: 222 days, 595 days), and for patients who had greater than or equal to $25 \%$ staining was 338.5 days (95\% Cl: 160 days, 731 days). Finally there was no statistical significance in survival time for the overall staining cohort ( $p<0.876)$. The estimated median time to survival for patients who had less than $25 \%$ staining was 385 days (95\% Cl: 183 days, 557 days), and for patients who had greater than or equal to $25 \%$ staining was 466 days (95\% Cl: 242 days, 595 days).

\section{Discussion}

\section{Spectrum of DAXX immunohistochemical staining in GBM}

The chromatin remodeler DAXX, seen as a predominantly nuclear protein, regulates the status of chromatin organization [6]. Historically DAXX is thought to be a predominantly nuclear stain [10]. Per contra our study found a more extensive spectrum of staining, where the percentage of cytoplasmic staining was about equal to that of nuclear staining. The mean for cytoplasmic staining percentage was $24.50 \%$ while the mean for nuclear staining percentage was $22.35 \%$.

DAXX, Death domain-associated protein, was first discovered through its cytoplasmic interaction with the classical death receptor Fas [6]. In the cytoplasm, the protein likely functions in regulating apoptosis [6]. Ergo it is not surprising that we found such voluminous staining in this region. This is the first study to demonstrate DAXX as a predominantly cytoplasmic stain in GBM.

\section{Role of DAXX in GBM tumorogenesis and tumor size}

Remarkably little is known about the role of DAXX in GBM despite numerous studies suggesting potential functions [11]. Research has shown that loss of DAXX results in the lengthening of telomeres (through a telomerase-independent process), resulting in alternate lengthening of telomeres (ALT) [12]. Subsequently ALT results in extensive genome rearrangements as well as defects in double-strand break repair [12]. Double-strand breaks are one of the most serious threats to the human genome, frequently leading to genomic instability and cancer [13]. This is the proposed mechanism of DAXX tumorigenesis in GBM.

In our study we were able to definitively prove that tumors with limited DAXX expression (defined as under $25 \%$ staining), were statistically larger. In most cohorts limited DAXX expression lead to tumors being at least one centimeter (or 25-30\%) larger. Other studies have shown that loss of nuclear ATRX and/or DAXX shows a near $100 \%$ correlation with the alternative lengthening of telomeres phenotype [14]. Research has shown that this likely results in problems in apoptosis, transcription, regulation of the p53 tumor suppressor pathway, and activity as a histone chaperone [11]. All of which promote tumor growth leading to larger cancers as seen in our study.

In GBM the standard of treatment is microsurgical resection with radiation and chemotherapy [15]. However gross total resection (GTR) can sometimes be limited from adhesions the tumor may have with surrounding anatomy [15]. Saying this GBM tumors that lack DAXX grow larger, have further adhesions and reduced possibility of gross total resection, as the feasibility and extent of surgical resection depends on tumor size [2]. With this in mind tumor size in GBM is an important predictor of prognosis adding to the significance of our study findings [2]. Unfortunately however Glioblastoma is disease without a cure despite extensive surgical resection [15].

Our findings promote the idea that DAXX likely plays a role in the tumorigenesis of adult GBM. This goes against what has traditionally been seen as a protein seen almost exclusively in pediatric GBM [16]. Future genome sequencing may identify a higher prevalence of DAXX mutations in adult patients than previously conceived.

\section{DAXX and its relationship to survival}

No studies have assessed the relationship between DAXX and prognosis in adult GBM. Our study detected a tendency for patients with definitive DAXX expression to live longer, however these results were not statistically significant. Possibly the reason that there was no statistically significant difference in survival is because the prognosis is always so poor, $2 \%$ of patients survive three years [2]. The overall prognosis for GBM has changed little since the 1980s [2]. This can make finding statistically significant prognostic variables very difficult in GBM. Patient survival depends on the following clinical and biologic parameters: tumor size, location, treatment, age at presentation Karnofsky performance score (KPS) at presentation, histologic findings, and molecular genetic factors [2]. Despite the fact that our study showed a statistical difference in tumor size we were unable to definitively prove the role of DAXX as a prognostic marker in adult GBM.

Currently research is being performed to establish whether targeting DAXX and components of its cellular pathways can be used as a therapeutic approach to treat childhood brain tumors $[17,18]$. Further research could bring about new drugs which target and potentially up-regulate DAXX in tumor cells without damaging healthy cells. For patients with GBM, this could lead to 
increased survival time and an improved prognosis. A notable example of how drug development can lead to increased survival in disease is that of chronic myeloid leukemia (CML) [19]. Following introduction of imatinib the estimated 8-year survival of CML improved from a historical rate of less than $20 \%$ up to $87 \%$ [19]. Future research regarding targeted DAXX therapies will possibly allow for better prognostic outcomes in patients with GBM.

\section{Role of DAXX expression in therapy decision mak- ing}

GBM exhibits a poor prognosis despite maximal multimodal therapy [16]. Patients with GBM commonly experience severe neurological symptoms, which increases the difficulty and risk of clinical treatment [20]. Often deciding what and how to treat patients with GBM can be challenging as the pros and cons of treatment methods must be considered [16].

There has ongoing research showing that the mitotic checkpoint protein is DAXX essential for responsiveness to taxanes, a widely used family of chemotherapeutics [21]. Paclitaxel, a taxane class drug induces apoptosis of glioblastoma cells via regulation apoptosis-related gene expression [21]. However it has been shown that absence or depletion of the protein DAXX increases cellular taxol resistance [21]. DAXX depletion increases cyclin B stability which subsequently results in taxol resistance (in mouse xenograft models) [21]. In one study Daxx depleted cells exhibited a strong taxolresistant phenotype with the majority of treated cells $(75-80 \%)$ capable of surviving after removal of taxol [21]. Per contra DAXX retained tumors treated with paclitaxel have been found to have significantly inhibited glioblastoma growth and prolonged survival in mice models [21].

The prognosis of patients with glioblastoma multiforme (GBM) remains poor despite advances in neurosurgery and radiotherapy $[19,22]$. Currently chemotherapy has done little to improve on these results $[19,22]$. Cranial irradiation remains the mainstay of treatment following surgery, however tumors almost always recur [22]. We feel strongly that chemotherapy could have a massive effect in the treatment of GBM. Currently adjuvant chemotherapy with temozolomide is the standard of care in patients with GBM aged $<70$ years [16]. There is a desperate need for more effective therapies in patients with GBM and taxol therapy could be one of them.

Throughout our research we have found no clincal trials that have treated DAXX un-mutated cohorts with taxol therapy. Research needs to be performed to separate treatment groups into DAXX mutated and DAXX non mutated groups. It is possible that patients without DAXX mutation and functional DAXX related apoptosis would benefit greatly from taxol therapy. Currently che- motherapeutics like taxols are not the standard of treatment of GBM $[19,22]$. There is potential for a clinical trial, as in our study, almost half of GBM tumors retained definite overall DAXX expression (33/72).

\section{Summary}

In summation our study found that DAXX was a predominantly cytoplasmic stain and is associated with tumor size in adult GBM. Statistically patient with tumors who had less than $25 \%$ immune-histochemical staining for DAXX had a significantly larger tumor size than patients who had greater than equal to $25 \%$ staining. Although tumor size is one of the biologic parameters associated with survival time, we were unable to definitively establish DAXX as a prognostic marker in GBM [2]. Prognostic studies in GBM are difficult as the disease is incurable and currently the prognosis is always poor.

Worth noting is that there is a potential role of DAXX in therapy decision making in GBM. As previously discussed tumors without DAXX mutations and ergo functional DAXX related apoptosis have been found to benefit greatly from taxol therapy in numerous studies [21]. Furthermore, research could bring about new drugs which target and potentially up-regulate DAXX in tumor cells without damaging healthy cells. This in turn could lead to increased survival time and prognosis.

In closing we feel that future genome sequencing may identify a higher prevalence of DAXX mutations in adult GBM than previously conceived. Ultimately future research will heighten our understanding of the role of DAXX in GBM, perhaps allowing targets to be tested for future therapies.

\section{Acknowledgments}

We thank Claudine Alexis and Kathy Quinn for their role as research coordinators.

\section{Disclosure}

The authors report no conflicts of interest in this work.

\section{References}

1. Davis ME (2016) Glioblastoma: Overview of disease and treatment. Clin J Oncol Nurs 20: 2-8.

2. Walid MS (2008) Prognostic factors for long-term survival after glioblastoma. Perm J 12: 45-48.

3. Pollack IF, Agnihotri S, Broniscer A (2019) Childhood brain tumors: Current management, biological insights, and future directions. J Neurosurg Pediatr 23: 261-273.

4. Benitez JA, Ma J, D'Antonio M, Boyer A, Camargo MF, et al. (2017) PTEN Regulates Glioblastoma Oncogenesis through Chromatin-Associated Complexes of DAXX and Histone H3.3. Nat Commun 8: 15223.

5. Louis DN, Perry A, Reifenberger G, von Deimling A, Figarella-Branger D, et al. (2016) The 2016 world health organization classification of tumors of the central nervous 
system: A summary. Acta Neuropathol 131: 803-820.

6. Yang X, Khosravi-Far R, Chang HY, Baltimore D (1997) Daxx, a novel Fas-binding protein that activates JNK and apoptosis. Cell 89: 1067-1076.

7. Xue Y, Gibbons R, Yan Z, Yang D, McDowell TL, et al. (2003) The ATRX syndrome protein forms a chromatin- remodeling complex with Daxx and localizes in promyelocytic leukemia nuclear bodies. Proc Natl Acad Sci U S A 100: 1063510640.

8. Schwartzentruber J, Korshunov A, Liu XY, Jones DT, Pfaff $\mathrm{E}$, et al. Driver mutations in histone $\mathrm{H} 3.3$ and chromatin remodelling genes in paediatric glioblastoma. Nature 482 226-231.

9. Jawhar NM (2009) Tissue microarray: A rapidly evolving diagnostic and research tool. Ann Saudi Med 29: 123-127.

10. Zizzi A, Montironi MA, Mazzucchelli R, Scarpelli M, LopezBeltran A, et al. (2013) Immunohistochemical analysis of chromatin remodeler DAXX in high grade urothelial carcinoma. Diagn Pathol 8: 111.

11. Amanda R Wasylishen, Jeannelyn S Estrella, Vinod Pant, Gilda P Chau, Guillermina Lozano (2018) Daxx functions are p53-independent in vivo. Molecular Cancer Research 16: 1523-1529.

12. Kallappagoudar S, Yadav RK, Lowe BR, Partridge JF (2015) Histone H3 mutations--a special role for H3.3 in tumorigenesis? Chromosoma 124: 177-189.

13. Xu Y, Her C (2014) DNA Double-strand break repair in tumorigenesis and anticancer treatment. Chemotherapy 3 : 124.

14. de Wilde RF, Heaphy CM, Maitra A, Meeker AK, Edil $\mathrm{BH}$, et al. (2012) Loss of ATRX or DAXX expression and concomitant acquisition of the alternative lengthening of telomeres phenotype are late events in a small subset of
MEN-1 syndrome pancreatic neuroendocrine tumors. Mod Pathol 25: 1033-1039.

15. Young RM, Jamshidi A, Davis G, Sherman JH (2015) Current trends in the surgical management and treatment of adult glioblastoma. Ann Transl Med 3: 121.

16. Minniti G, Muni R, Lanzetta G, Marchetti P, Enrici RM (2009) Chemotherapy for glioblastoma: Current treatment and future perspectives for cytotoxic and targeted agents. Anticancer Res 29: 5171-5184.

17. Haase S, Garcia-Fabiani MB, Carney S, Altshuler D, Núñez FJ, et al. (2018) Mutant ATRX: Uncovering a new therapeutic target for glioma. Expert Opin Ther Targets 22: 599-613.

18. Fan HC, Chen CM, Chi CS, Tsai JD, Chiang KL, et al. (2019) Targeting telomerase and ATRX/DAXX inducing tumor senescence and apoptosis in the malignant glioma. Int J Mol Sci 20: 200.

19. Kantarjian H, O'Brien S, Jabbour E, Garcia-Manero G, Quintas-Cardama A, et al. (2012) Improved survival in chronic myeloid leukemia since the introduction of imatinib therapy: A single-institution historical experience. Blood 119: 1981-1987.

20. Vecht CJ, Kerkhof M, Duran-Pena A (2014) Seizure prognosis in brain tumors: New insights and evidencebased management. Oncologist 19: 751-759.

21. Giovinazzi S, Lindsay CR, Morozov VM, Escobar-Cabrera E, Summers MK, et al. (2012) Regulation of mitosis and taxane response by Daxx and Rassf1. Oncogene 31: 13-26.

22. Whitehead CA, Nguyen HPT, Morokoff AP, Luwor RB, Paradiso L, et al. (2018) Inhibition of radiation and temozolomide-induced invadopodia activity in glioma cells using FDA-approved drugs. Transl Oncol 11: 1406-1418. 